\title{
Transcriptome Sequencing Reveals High-Salt Diet- Induced Abnormal Liver Metabolic Pathways in Mice
}

\section{Yanping Li}

Department of Gastroenterology, Civil Aviation General Hospital

\section{Yufei Lyu}

Beijing Institute of Biotecnology

Jing Huang

Beijing Institute of Biotecnology

Kun Huang ( $\nabla$ hkunmed@126.com )

Department of Gastroenterology, Civil Aviation General Hospital

Jiufei Yu

Department of Gastroenterology, Civil Aviation General Hospital

\section{Research Article}

Keywords: high-salt diet, transcriptome sequencing, liver

Posted Date: January 13th, 2021

DOI: https://doi.org/10.21203/rs.3.rs-140557/v1

License: (a) (i) This work is licensed under a Creative Commons Attribution 4.0 International License. Read Full License 


\section{Abstract}

Background: Although salt plays an important role in maintaining the normal physiological metabolism of the human body, many potential abnormalities in liver, especially with normal pathological results, caused by high-salt diet are not well characterized.

Methods: Eight-week-old female C57BL/ 6 mice were randomly divided into a normal group and a high salt group. After feeding with normal or sodium-rich chow (containing $6 \% \mathrm{NaCl}$ ) for 6 weeks. Liver injury were evaluated and the influences of a high-salt diet on liver were analyzed by transcriptome sequencing.

Results: We found that although no liver parenchymal injury was found after high-salt feeding, many metabolic abnormalities had formed based on transcriptome sequencing results. GO and KEGG enrichment analyses of differentially expressed genes revealed that at least 15 enzyme activities and the metabolism of multiple substances were affected by a high-salt diet. Moreover, a variety of signaling, and metabolic pathways, as well as biological functions, including some known pathways and many novel ones, such as retinol metabolism, linoleic acid metabolism, steroid hormone biosynthesis, and signaling pathways, were involved in liver dysfunction due to a high-salt diet.

Conclusions: High-salt diet could induce a serious abnormal liver metabolic pathway in mice, although substantial damage has not yet been shown. This study is the first to reveal the impact of a high-salt diet on the liver at the omics level, and provides theoretical support for potential clinical risk evaluation, pathogenic mechanisms, and drug design for combating liver dysfunction, and this study also provides a serious candidate direction for further research on the physiological impacts of high-salt diets.

\section{Background}

As an essential condiment in daily life, salt plays an important role in maintaining the normal physiological metabolism of the human body. Although the World Health Organization recommends that the daily intake of salt should not exceed $5 \mathrm{~g}$, the daily intake of salt is often greater than $10 \mathrm{~g}$ [1]. This is particularly true in some countries and regions where salt intake is often higher due to dietary habits [2], which can lead to some potential disease risks in the populations. Studies have found that a long-term high-salt diet results in potential harm to the body, inducing cardiovascular disease [3, 4], insulin resistance and type 2 diabetes [5], metabolic syndrome, obesity, muscle atrophy [6-8], and problems with the immune system [9].

Some mechanisms of organ damage caused by high salt intake have been elucidated thus far. For example, animal experiments have revealed that a high-salt diet can cause kidney damage through the reduction of ACE2, enhancement of leukocyte adhesion, blunt renal autoregulation via a reactive oxygen species-dependent mechanism, etc. [10-12]. A high-salt diet can also cause cardiovascular abnormalities and high plasma sodium can cause hypertension by direct effects on endothelial functions, thus controlling vascular tone [13], or by participating in cardiovascular injury through hormonal pathways [14]. In addition, a high-salt diet also has direct or indirect effects on the liver and cause diseases like 
fibrosis and fatty liver $[15,16]$. Although many mechanisms of liver damage caused by high-salt diets have been revealed to date, the liver, as the largest metabolic organ, is the site of a variety of important signaling pathways; whether these are affected by a high-salt diet and are part of the cause of specific diseases is still unclear.

With the development of transcriptome sequencing technology in recent years, it has become feasible to analyze all mRNA transcribed by a specific cell or organ in a certain functional state [17-19]. At present, transcriptome sequencing has been applied to many medical fields, such as clinical diagnosis, marker screening, prognosis evaluation, and pathogenesis [20-22].

In this study, we sequenced the liver transcriptome of normal and high-salt diet mice, and explored the effects of a high salt diet on the liver at a gene expression level. In addition to some abnormal signaling and metabolic pathways that have been reported previously, we also identified many new abnormal metabolic pathways, which provides a strong theoretical basis for the potential clinical risks of a high-salt diet, the diseases and pathogenic mechanisms it correlates with, and provides targets for a rational drug design to treat high-salt diet-induced liver dysfunction.

\section{Methods}

\section{Animals}

Eight-week-old female C57BL/6 mice, 16-19 g, were purchased from Charles River, Beijing, China, and housed in the Laboratory Animal Center of the Academy of Military Medical Sciences, Beijing, China. The mice were randomly divided into a normal group and a high salt group. The normal group was fed with normal chow, and the high salt group was fed with sodium-rich chow (containing $6 \% \mathrm{NaCl}$ ) for 6 weeks.

\section{Hematoxylin-Eosin staining (H\&E staining)}

H\&E staining was performed according to the instructions of a Hematoxylin-Eosin Staining Kit (Solarbio, China, \#G1121). Briefly, after dissection, the liver tissues of mice were fixed with a $4 \%$ fixative solution, embedded in paraffin, and then sectioned. The paraffin sections were washed twice with xylene for $10 \mathrm{~min}$, and then washed sequentially with absolute ethanol, $95 \%$ ethanol, $80 \%$ ethanol, $70 \%$ ethanol, and distilled water for two minutes. Next, sections were stained with hematoxylin for 5 min and washed with water for $8 \mathrm{~s}$. The sections were then incubated in differentiation solution for $5 \mathrm{~s}$ and again washed with water for $30 \mathrm{~s}$. After incubation in blue returning solution for $1 \mathrm{~min}$, the sections were rinsed with water for $30 \mathrm{~s}$ and eosin-stained for $1 \mathrm{~min}$. The sections were then washed with water, $80 \%$ ethanol, $90 \%$ ethanol, $95 \%$ ethanol, $95 \%$ ethanol, absolute ethanol for $5 \mathrm{~s}$ each and then washed with absolute ethanol and xylene for 1 min each. Finally, they were fixed with neutral balata.

\section{RNA extraction}

After mice were euthanized, liver tissues were removed and RNAs were extracted according to the instructions of the Total RNA Extraction Kit (Solarbio, China, \#R1200). 


\section{Construction of transcriptome libraries}

First, mature mRNAs were isolated from total RNA using oligo (dT) magnetic beads, and these were then randomly fragmented by mixing with fragmentation buffer. Then, first strand cDNAs were synthesized using mRNAs as templates and second strand cDNAs were synthesized by adding PCR buffer, dNTPs, RNase $H$, and DNA polymerase I. After purification, the double strand cDNAs were resolved with EB buffers for end repair and A-tailing, and then the target fragments were recovered by agarose gel electrophoresis and the final libraries were obtained by PCR amplification. After passing library quality inspection, an Illumina platform was used to carry out high-throughput sequencing.

\section{Quality control of sequencing data}

First, the data were filtered by removing contaminated sequences, low-quality sequences, and sequences containing more than $5 \% \mathrm{n}$. Then, the mass distribution and base distribution were analyzed and compared to ensure the reliability of subsequent analysis. Finally, the filtered sequences from each sample were compared with a mouse reference genome.

\section{Statistical analysis}

Data were expressed as mean \pm standard deviations, and statistical analyses were completed using GraphPad 8.0. A Student's t-test was used to compare the difference between two groups and differences were considered statistically significant at $p<0.05$.

\section{Results}

\section{Effects of a high-salt diet on liver function in mice}

Although there have been many reports on the effects of long-term high-salt diets on the liver, salt, as a common flavoring agent, is ubiquitous in cuisine, and may cause this damage to be very chronic and long-term over an average human's lifespan. In order to evaluate whether liver function could be affected by 6 weeks of a high-salt $(6 \% \mathrm{NaCl})$ diet, biochemical indexes including alanine aminotransferase activity (ALT), glutamic oxaloacetic transaminase activity (AST), and alkaline phosphatase activity (ALP) in blood serum were detected after 6 weeks of feeding. The results showed that there were no significant differences in these biochemical indices between normal and high-salt diet mice (Fig. 1a). Moreover, HDE staining of liver tissue also showed no significant pathological changes after 6 weeks of high-salt feeding (Fig. 1b), although the central vein of the hepatic lobule in the high-salt diet group was enlarged. These results indicated that a high-salt diet, on the surface, did not cause significant damage to the liver over this timeframe.

\section{Overall changes in RNA expression in liver tissue with a high-salt diet}


Although, at the macro level, we did not find clear evidence of the effect of a high-salt diet on the liver, the potential effects of this diet on the liver may be more subtle. Therefore, we extracted RNA from the livers of mice raised for six weeks with a normal or high-salt diet, and used transcriptome sequencing to further analyze the effects of a high-salt diet on liver. After sequencing, $6 \mathrm{~Gb}$ of data from each sample was obtained, and after filtering for original sequences, the high-quality sequences obtained from the normal and high-salt groups were on average $46.02 \mathrm{Mb}$ and $44.68 \mathrm{Mb}$ in contiguous length, respectively. The matching rates to a reference genome were $97.43 \%$ and $97.44 \%$, respectively, and the total number of genes detected was 18,916 and 19,204, respectively (Table S1). In order to evaluate the overall trend of sample expression more objectively, we converted the fragments from each gene to Fragments Per Kilobase per Million (FPKM) mapped fragments. Since the number of differentially expressed genes only accounts for a small part of the overall number of genes and a small number of differentially expressed genes had no significant effect on the expression distribution of our samples, the overall distribution of the samples from the box diagram was basically the same when we compared each group (Fig. 2). These results indicated that our data met requirements in quality and could be used for further analysis.

\section{Analysis of liver differential genes by GO and KEGG enrichment}

During the identification of differentially expressed genes, a fold change $\geq 2$ and a padj value (corrected $p$ value) $<0.05$ were used as the screening criteria, and we found that there were 52 differential genes, including 33 up-regulated genes and 19 down-regulated genes. For example, some up-regulated genes like cyp4a10 and cyp4a14, which are related to the formation of non-alcoholic fatty liver (NAFLD), and rad51b, which is related to DNA repair, and some down-regulated genes like cyp17a1, which is involved in the synthesis of steroid hormones, and cyp2a4, which is involved in many metabolisms of drugs and compounds, made logical sense based on the previous literature (Fig. 3).

In order to further analyze the function of these differential genes, Gene ontology (GO) analysis was performed. According to the secondary items in the $\mathrm{GO}$ database, the number of differentially expressed genes in these items were counted, and percentages were calculated. The results showed that in the 36 sections of three main categories (biological process, cellular component and molecular function), extracellular protein, macromolecular complex, cell killing, immune process, cell proliferation, cell growth, and cell movement were all significantly up-regulated in the high-salt diet group (Fig. 4).

On the basis of the differentially expressed genes in each GO entry, we further analyzed these entries in terms of significantly enriched differentially expressed genes compared with the whole genome background. The results yielded 25 significantly different terms, including 2 terms in the biological process category (Fig. 5a) and 23 terms in the molecular function category (Fig. 5b and Table S2). Specifically, we found that at least 15 enzyme activities and the metabolism of multiple substances were involved. The abnormal activities of these enzymes and metabolisms were associated with many diseases. 
Next, Kyoto encyclopedia of genes and genomes (KEGG) enrichment analysis showed that the differentially expressed genes induced by high-salt diet were involved in 33 pathways, including some previously reported pathways, like the PPAR signaling pathway [23, 24], the MAPK signaling pathway [25], and the prolactin signaling pathway [26], and some that have not yet been reported, such as the PI3K-Akt signaling pathway, retinol metabolism, and steroid hormone biosynthesis (Table 1). These pathways represent potential risks for some diseases. 
Table 1

KEGG result between high-salt group and normal group

\begin{tabular}{|c|c|c|}
\hline KEGG pathways & KEGG map number & $p$ \\
\hline PPAR signaling pathway & map03320 & 0.0087 \\
\hline MAPK signaling pathway & map04010 & 0.3900 \\
\hline Prolactin signaling pathway & map04917 & 0.1117 \\
\hline PI3K-Akt signaling pathway & map04151 & 0.5366 \\
\hline TGF-beta signaling pathway & map04350 & 0.0095 \\
\hline Inflammatory mediator regulation of TRP channels & map04750 & 0.0182 \\
\hline Signaling pathways regulating pluripotency of stem cells & map04550 & 0.0210 \\
\hline Aldosterone synthesis and secretion & map04925 & 0.1241 \\
\hline Metabolism of xenobiotics by cytochrome P450 & map00980 & 0.0083 \\
\hline Drug metabolism - cytochrome P450 & map00982 & 0.1196 \\
\hline Drug metabolism - other enzymes & map00983 & 0.0980 \\
\hline Linoleic acid metabolism & map00591 & 0.0876 \\
\hline Ascorbate and aldarate metabolism & map00053 & 0.0435 \\
\hline Porphyrin and chlorophyll metabolism & map00860 & 0.0604 \\
\hline Tryptophan metabolism & map00380 & 0.0616 \\
\hline Retinol metabolism & map00830 & 0.0000 \\
\hline Arachidonic acid metabolism & map00590 & 0.0106 \\
\hline Steroid hormone biosynthesis & map00140 & 0.0004 \\
\hline Ovarian steroidogenesis & map04913 & 0.0980 \\
\hline Pentose and glucuronate interconversions & map00040 & 0.0864 \\
\hline Fatty acid degradation & map00071 & 0.0031 \\
\hline Ubiquitin mediated proteolysis & map04120 & 0.4150 \\
\hline Endocytosis & map04144 & 0.4456 \\
\hline Vascular smooth muscle contraction & map04270 & 0.0184 \\
\hline Bile secretion & map04976 & 0.0060 \\
\hline Salivary secretion & map04970 & 0.1083 \\
\hline Homologous recombination & map03440 & 0.0688 \\
\hline
\end{tabular}




\begin{tabular}{|lll|}
\hline KEGG pathways & KEGG map number & $\boldsymbol{p}$ \\
\hline Epstein-Barr virus infection & map05169 & 0.3673 \\
\hline Peroxisome & map04146 & 0.1331 \\
\hline Phagosome & map04145 & 0.2999 \\
\hline Tight junction & map04530 & 0.3439 \\
\hline Transcriptional misregulation in cancer & map05202 & 0.3035 \\
\hline Chemical carcinogenesis & map05204 & 0.0007 \\
\hline
\end{tabular}

\section{Discussion}

As the largest metabolic organ, the liver plays an important role in the health of the body, including three major nutrients and various molecular metabolic events, and this organ hosts some key nodes. Thus, some abnormalities will have potential impacts on health. We compared the transcriptome changes in the liver of mice fed with high-salt diet by transcriptome sequencing and found that there were many abnormal signal pathways and metabolic events in the liver after exposure to a high-salt diet. For example, two up-regulated genes, cyp4a10 and cyp4a14, were shown to be involved in the PPAR signaling pathway and related to oxidative stress and lipid peroxidation of fatty acids, causing NAFLD/steatohepatitis (NASH). In steroid hormone biosynthesis pathways, the cyp17a1 gene was downregulated, and in cell proliferation and apoptosis regulation pathways, nr4a1 gene expression was downregulated. Many of the pathways we identified here have been previously reported. In addition, we also found some other potential effects, such as retinol metabolism, ascorbate and aldarate metabolism, and steroid hormone biosynthesis, which have not been reported previously and can provide mechanistic support for disease prediction of those caused by a high-salt diet.

It has been reported that long term high-salt diet can directly cause liver fibrosis, or lead to liver fat accumulation, suggesting lipoprotein transport disorders are caused by a high-salt diet [16, 27]. In addition, studies have shown that a high-salt intake increases liver osmotic pressure, promotes the expression of the transcription factor TonEBP, and then activates the expression of aldose reductase, promoting the production of endogenous fructose. Therefore, salt may be a potential cause of obesity and metabolic syndrome [15]. Accordingly, a study also found that the prevalence of NAFLD increased with an increase in $\mathrm{Na}^{+}$intake, implying the effect of a high-salt diet on liver lipid metabolism and the potential relationship between NAFLD and obesity [28]. Although our results showed that after 6 weeks of high-salt diet, biochemical and H口E staining showed no obvious liver damage (probably due to the short time of high-salt feeding, where organic damage had not yet formed), sequencing results provided clues to the potential influences which need to be further researched.

There is a significant correlation between high salt diet and hypertension. A large amount of evidence has shown that salt is the main cause of blood pressure elevation, and a decrease in salt intake reduces 
blood pressure, thus reducing the diseases related to blood pressure. The central mechanism of hypertension shows that a high-salt diet leads to an increase of the brain's sodium ion content, which activates the sympathetic nerves through the $\mathrm{Na}(+)$-ENaC-RAAS-EDLF axis and promotes the formation of hypertension [29]. It has also been found that salt may induce salt-sensitive hypertension by inhibiting the expression of renal enzymes [30]. In addition, high sodium levels can directly promote the proliferation of vascular smooth muscle cells and promote the formation of hypertension [31]. Our results also showed that the expression of the cyp4a gene in the liver was significantly increased, while Cyp4a protein could hydroxylate arachidonic acid into 20-hydroxyeicosapentaenoic acid and act on blood vessels, which indirectly participate in the formation of hypertension. This finding also supported a previous mechanism of a high-salt diet leading to hypertension [32].

With the continuous development and popularization of omics research technology (including proteome, transcriptome, and metabolomic) over the past few years, people have gained the ability to systematically describe and analyze the changes of specific levels of the body as a whole, so as to further explore the pathogenesis of diseases, allowing them to identify the potential pathogenic risks and influencing factors for these diseases. Among these technologies, transcriptome sequencing technology is an important means to study the pathogenesis of many diseases. Since transcription levels are often positively correlated with protein expression levels, transcriptome sequencing can be used to analyze and predict the occurrence and development of diseases. By comparing and analyzing the differential genes under different conditions in the transcriptional phase, we can identify changes that may be related to diseases. We also could analyze the correlation between various pathways, thus providing clues to the pathogenesis of diseases. This study systematically analyzed the liver after high-salt diet at the transcriptomics level, and we found many potential risks for diseases, which provide clues to the pathogenesis, prevention, and drug target research of diseases. These potential pathogenic factors will be further explored in the future.

\section{Conclusions}

Our study has indicated that a long-term high-salt diet has many potential effects on the liver. Although substantial damage has not yet been shown, we found that a high-salt diet could influence at least 15 enzyme activities and the metabolism of multiple substances. In addition, except for some pathways consistent with some known mechanisms of damages caused by a high-salt diet, we also found that a long-term high-salt diet has an impact on other important pathways in the liver. This study is the first to reveal the impact of a high-salt diet on the liver at the omics level, and provides theoretical support for potential clinical risk evaluation, pathogenic mechanisms, and drug design for the treatment of liver dysfunction. Furthermore, it provides a serious candidate direction for the research of high-salt diets.

\section{Abbreviations}


alanine aminotransferase activity; AST:glutamic oxaloacetic transaminase activity; ALP:alkaline phosphatase activity; FPKM:Fragments Per Kilobase per Million; NAFLD:non-alcoholic fatty liver; GO:Gene ontology; KEGG:Kyoto encyclopedia of genes and genomes.

\section{Declarations}

Ethics approval and consent to participate: All of the animal experiments were approved by and performed in accordance with the recommendations of the Academy of Military Medical Sciences Institutional Animal Care and Use Committee (IACUC-DWZX-2020-064). Animals were housed with a constant ambient temperature $\left(23 \pm 3^{\circ} \mathrm{C}\right)$ and humidity $(55 \% \pm 5 \%)$. Food, bedding, and water were changed every 4 days. All efforts were made to minimize suffering.

Competing interests: The authors declare no conflict of interest.

Availability of data and materials: The datasets generated and/or analyzed during the current study are available in the GEO DataSets, https://www.ncbi.nlm.nih.gov/geo/query/acc.cgi?acc=GSE163294

Consent for publication: Not applicable.

Authors' contributions: Conducted the experiments: LY, LY, HJ. Processed the experimental data: LY, HJ, HK. Performed the theoretical analyses: LY, HK, YJ. Written the manuscript: LY, HK, YJ. All the authors commented on drafts and have approved the final version of the manuscript.

Funding: This research was funded by Foundation of Civil Aviation General Hospital (Civil Aviation Medical Center), grant number 201920.

Acknowledgements: Not applicable.

\section{References}

1. Brown IJ, Tzoulaki I, Candeias V, Elliott P. Salt intakes around the world: implications for public health. International journal of epidemiology 2009; 38(3):791-813.

2. Zhang J, Guo X, Lu Z, Tang J, Li Y, Xu A, Liu S. Cardiovascular Diseases Deaths Attributable to High Sodium Intake in Shandong Province, China. Journal of the American Heart Association 2019; 8(1):e010737.

3. Bibbins-Domingo K, Chertow GM, Coxson PG, Moran A, Lightwood JM, Pletcher MJ, Goldman L. Projected effect of dietary salt reductions on future cardiovascular disease. The New England journal of medicine 2010; 362(7):590-599.

4. Li Y, Huang Z, Jin C, Xing A, Liu Y, Huangfu C, Lichtenstein AH, Tucker KL, Wu S, Gao X. Longitudinal Change of Perceived Salt Intake and Stroke Risk in a Chinese Population. Stroke 2018; 49(6):13321339. 
5. Vedovato M, Lepore G, Coracina A, Dodesini AR, Jori E, Tiengo A, Del Prato S, Trevisan R. Effect of sodium intake on blood pressure and albuminuria in Type 2 diabetic patients: the role of insulin resistance. Diabetologia 2004; 47(2):300-303.

6. Larsen SC, Angquist L, Sorensen TI, Heitmann BL. 24 h urinary sodium excretion and subsequent change in weight, waist circumference and body composition. PloS one 2013; 8(7):e69689.

7. Libuda L, Kersting M, Alexy U. Consumption of dietary salt measured by urinary sodium excretion and its association with body weight status in healthy children and adolescents. Public health nutrition 2012; 15(3):433-441.

8. Huh JH, Lim JS, Lee MY, Chung CH, Shin JY. Gender-specific association between urinary sodium excretion and body composition: Analysis of the 2008-2010 Korean National Health and Nutrition Examination Surveys. Metabolism: clinical and experimental 2015; 64(7):837-844.

9. Jobin K, Stumpf NE, Schwab S, Eichler M, Neubert P, Rauh M, Adamowski M, Babyak O, Hinze D, Sivalingam $S$ et al. A high-salt diet compromises antibacterial neutrophil responses through hormonal perturbation. Science translational medicine 2020; 12(536).

10. Bernardi S, Toffoli B, Zennaro C, Tikellis C, Monticone S, Losurdo P, Bellini G, Thomas MC, Fallo F, Veglio $\mathrm{F}$ et al. High-salt diet increases glomerular ACE/ACE2 ratio leading to oxidative stress and kidney damage. Nephrology, dialysis, transplantation: official publication of the European Dialysis and Transplant Association - European Renal Association 2012; 27(5):1793-1800.

11. Takahashi H, Nakagawa S, Wu Y, Kawabata Y, Numabe A, Yanagi Y, Tamaki Y, Uehara Y, Araie M. A high-salt diet enhances leukocyte adhesion in association with kidney injury in young Dahl saltsensitive rats. Hypertension research: official journal of the Japanese Society of Hypertension 2017; 40(11):912-920.

12. Fellner RC, Cook AK, O'Connor PM, Zhang S, Pollock DM, Inscho EW. High-salt diet blunts renal autoregulation by a reactive oxygen species-dependent mechanism. American journal of physiology Renal physiology 2014; 307(1):F33-40.

13. Oberleithner H, Riethmuller C, Schillers H, MacGregor GA, de Wardener HE, Hausberg M. Plasma sodium stiffens vascular endothelium and reduces nitric oxide release. Proceedings of the National Academy of Sciences of the United States of America 2007; 104(41):16281-16286.

14. Fedorova OV, Shapiro JI, Bagrov AY. Endogenous cardiotonic steroids and salt-sensitive hypertension. Biochimica et biophysica acta 2010; 1802(12):1230-1236.

15. Lanaspa MA, Kuwabara M, Andres-Hernando A, Li N, Cicerchi C, Jensen T, Orlicky DJ, RoncalJimenez CA, Ishimoto T, Nakagawa T et al. High salt intake causes leptin resistance and obesity in mice by stimulating endogenous fructose production and metabolism. Proceedings of the National Academy of Sciences of the United States of America 2018; 115(12):3138-3143.

16. Wang G, Yeung CK, Wong WY, Zhang N, Wei YF, Zhang JL, Yan Y, Wong CY, Tang JJ, Chuai M et al. Liver Fibrosis Can Be Induced by High Salt Intake through Excess Reactive Oxygen Species (ROS) Production. Journal of agricultural and food chemistry 2016; 64(7):1610-1617. 
17. Lockhart DJ, Winzeler EA. Genomics, gene expression and DNA arrays. Nature 2000; 405(6788):827836.

18. Metzker ML. Sequencing technologies - the next generation. Nature reviews Genetics 2010; 11(1):3146.

19. Ozsolak F, Milos PM. RNA sequencing: advances, challenges and opportunities. Nature reviews Genetics 2011; 12(2):87-98.

20. Byron SA, Van Keuren-Jensen KR, Engelthaler DM, Carpten JD, Craig DW. Translating RNA sequencing into clinical diagnostics: opportunities and challenges. Nature reviews Genetics 2016; 17(5):257-271.

21. Sinicropi D, Qu K, Collin F, Crager M, Liu ML, Pelham RJ, Pho M, Dei Rossi A, Jeong J, Scott A et al. Whole transcriptome RNA-Seq analysis of breast cancer recurrence risk using formalin-fixed paraffinembedded tumor tissue. PloS one 2012; 7(7):e40092.

22. Patino LH, Ramirez JD. RNA-seq in kinetoplastids: A powerful tool for the understanding of the biology and host-pathogen interactions. Infection, genetics and evolution: journal of molecular epidemiology and evolutionary genetics in infectious diseases 2017; 49:273-282.

23. Gao F, Liang Y, Wang X, Lu Z, Li L, Zhu S, Liu D, Yan Z, Zhu Z. TRPV1 Activation Attenuates High-Salt Diet-Induced Cardiac Hypertrophy and Fibrosis through PPAR-delta Upregulation. PPAR research 2014; 2014:491963.

24. Ozurumba E, Mathew O, Ranganna K, Choi M, Oyekan A. Regulation of hypoxia inducible factor/prolyl hydroxylase binding domain proteins 1 by PPARalpha and high salt diet. Journal of basic and clinical physiology and pharmacology 2018; 29(2):165-173.

25. Miranda PM, De Palma G, Serkis V, Lu J, Louis-Auguste MP, McCarville JL, Verdu EF, Collins SM, Bercik P. High salt diet exacerbates colitis in mice by decreasing Lactobacillus levels and butyrate production. Microbiome 2018; 6(1):57.

26. Fressinaud P, Rohmer V, Galland F, Marcais J, Bigorne JC, Fressinaud L. [Effects of low, normal and high sodium diet on antidiuretic hormone and prolactin (author's transl)]. Annales d'endocrinologie 1980; 41(1):63-64.

27. Huh JH, Lee KJ, Lim JS, Lee MY, Park HJ, Kim MY, Kim JW, Chung CH, Shin JY, Kim HS et al. High Dietary Sodium Intake Assessed by Estimated 24-h Urinary Sodium Excretion Is Associated with NAFLD and Hepatic Fibrosis. PloS one 2015; 10(11):e0143222.

28. Choi Y, Lee JE, Chang Y, Kim MK, Sung E, Shin H, Ryu S. Dietary sodium and potassium intake in relation to non-alcoholic fatty liver disease. The British journal of nutrition 2016; 116(8):1447-1456.

29. Takahashi $H$, Yoshika M, Komiyama Y, Nishimura M. The central mechanism underlying hypertension: a review of the roles of sodium ions, epithelial sodium channels, the renin-angiotensinaldosterone system, oxidative stress and endogenous digitalis in the brain. Hypertension research: official journal of the Japanese Society of Hypertension 2011; 34(11):1147-1160.

30. Zheng WL, Wang J, Mu JJ, Liu FQ, Yuan ZY, Wang Y, Wang D, Ren KY, Guo TS, Xiao HY. Effects of salt intake and potassium supplementation on renalase expression in the kidneys of Dahl salt- 
sensitive rats. Experimental biology and medicine 2016; 241(4):382-386.

31. Wu Y, Zhou J, Wang H, Wu Y, Gao Q, Wang L, Zhao Q, Liu P, Gao S, Wen W et al. The activation of p38 MAPK limits the abnormal proliferation of vascular smooth muscle cells induced by high sodium concentrations. International journal of molecular medicine 2016; 37(1):74-82.

32. Williams JM, Murphy S, Burke M, Roman RJ. 20-hydroxyeicosatetraeonic acid: a new target for the treatment of hypertension. Journal of cardiovascular pharmacology 2010; 56(4):336-344.

\section{Figures}

a

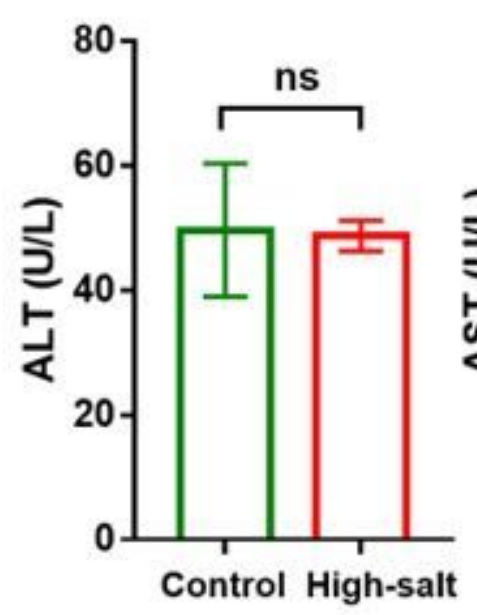

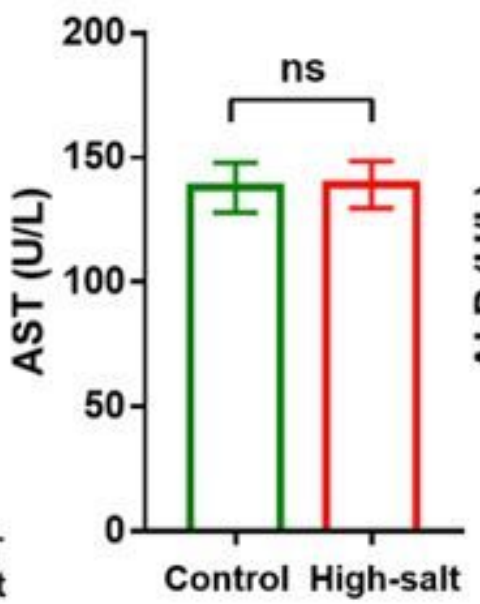

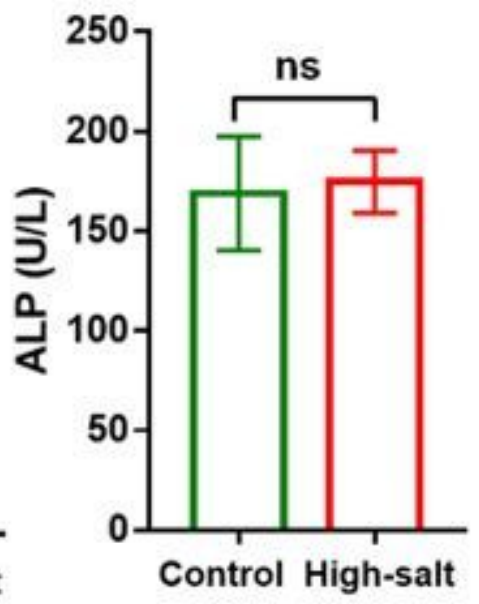

b
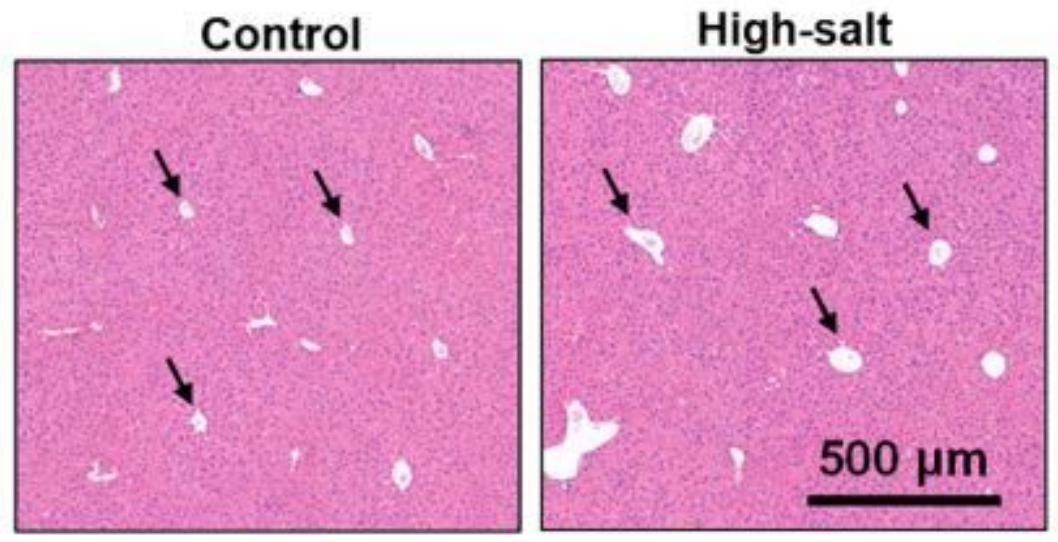

\section{Figure 1}

The effect of a high-salt diet on liver function: (a) ALT, AST, and ALP in blood serum after 6 weeks of feeding $(n=5)$. ns indicates no significant difference; (b) $H \otimes E$ staining of liver tissue after 6 weeks. The arrows point to the central vein of hepatic lobule. 


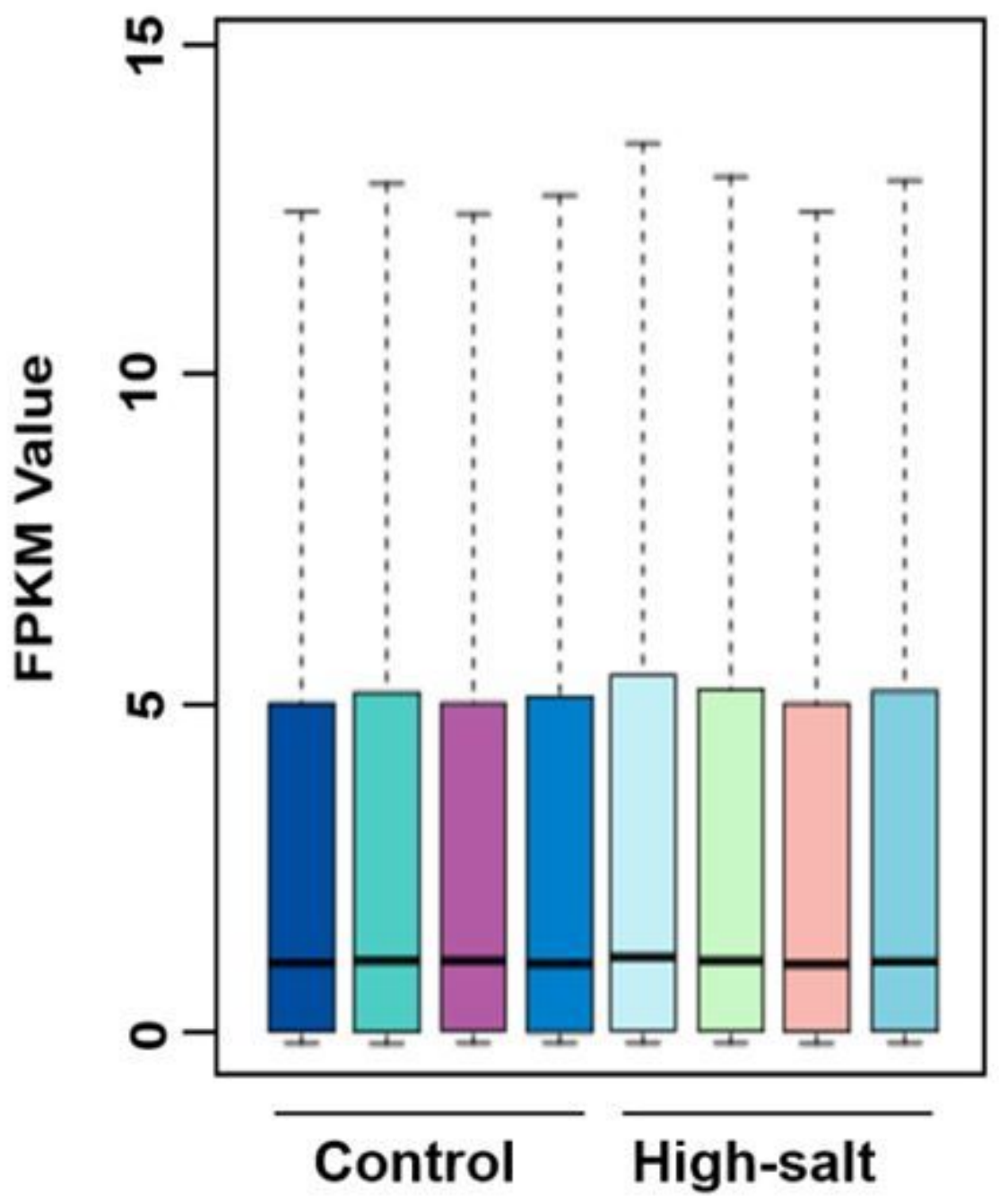

Figure 2

Overall distribution of gene expression in each sample. The expression of each sample was analyzed and is shown with a box diagram. The FPKM value indicates the logarithm of the gene expression from each sample. The bar indicates the range from maximum to minimum. The column indicates the range from upper quartile to lower quartile. The line in each column indicates the median. 


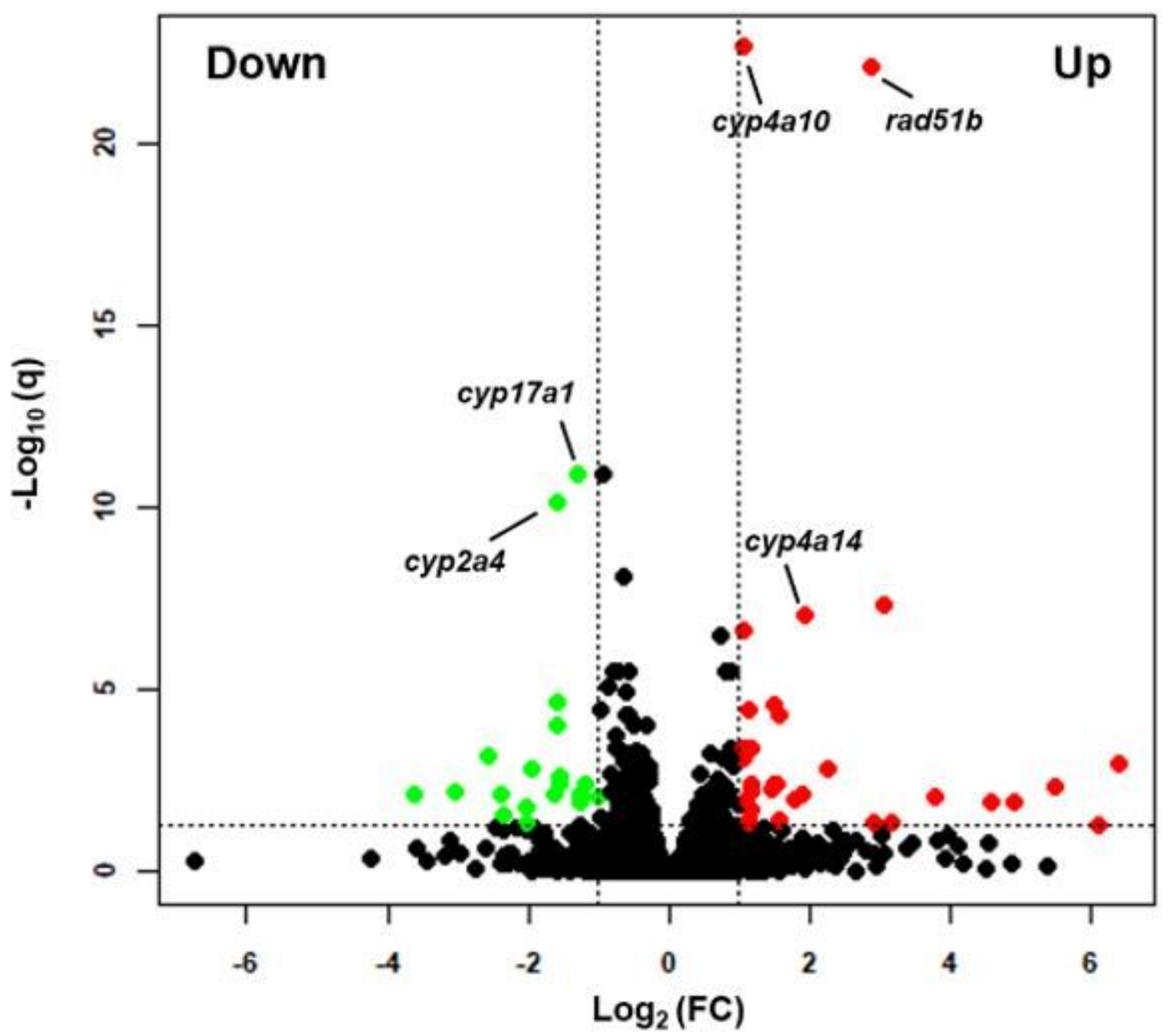

Figure 3

Differentially expressed genes in high-salt diet. The expressed genes were compared between normal and high-salt diet group, and the differences in the gene expression levels and statistical significance between these two groups were further illustrated by volcano plot. FC indicates the fold change compared with the control group. The green points indicate up-regulated genes and red points indicate down-regulated genes. 


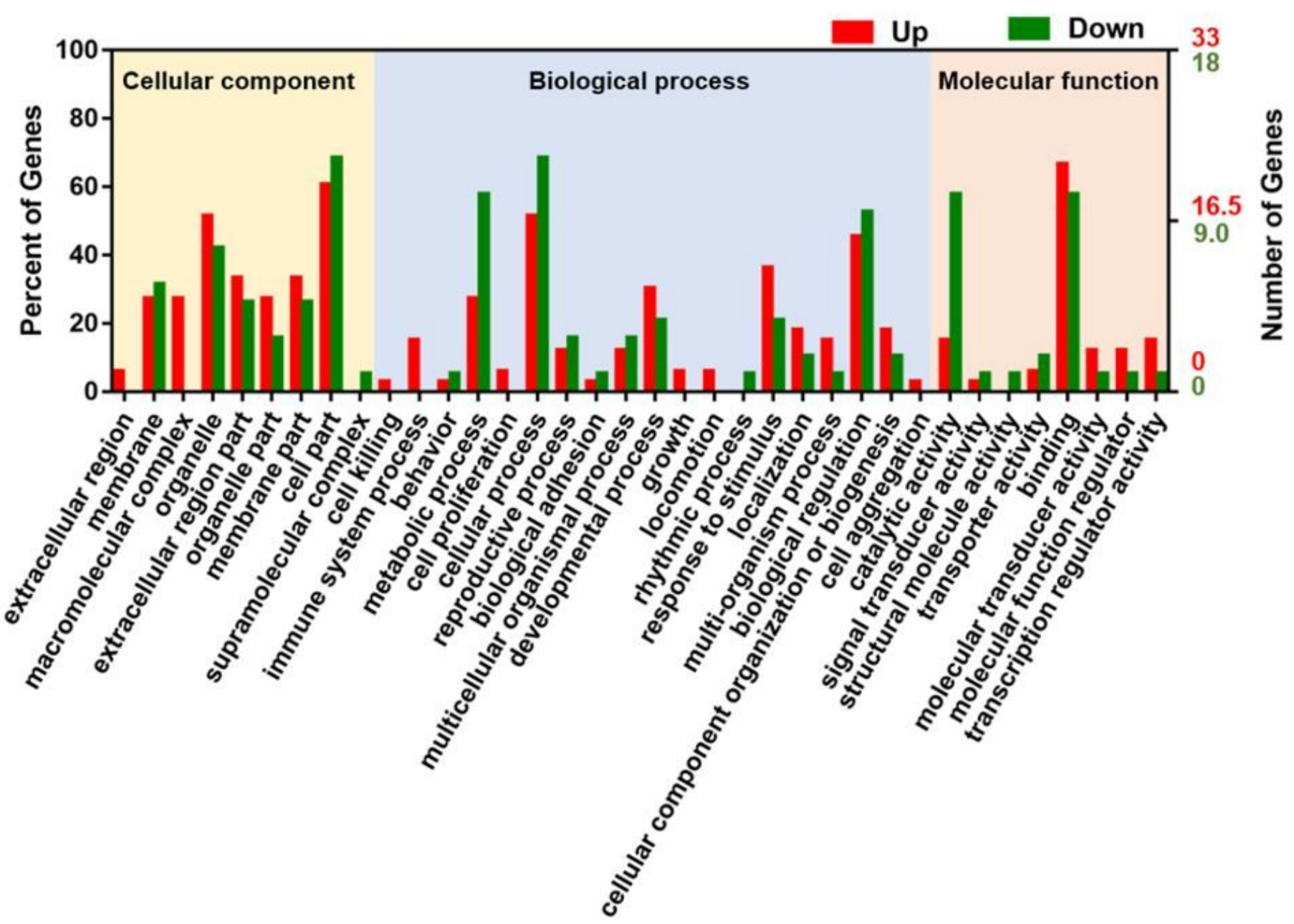

Figure 4

GO enrichment of high-salt diet-induced differentially expressed gene sets. GO enrichment analysis of differentially expressed genes was performed and the differentially expressed genes were enriched in 36 sections of the 3 main categories (biological process, cellular component and molecular function). Up indicates up-regulated terms and Down indicates down-regulated terms. 

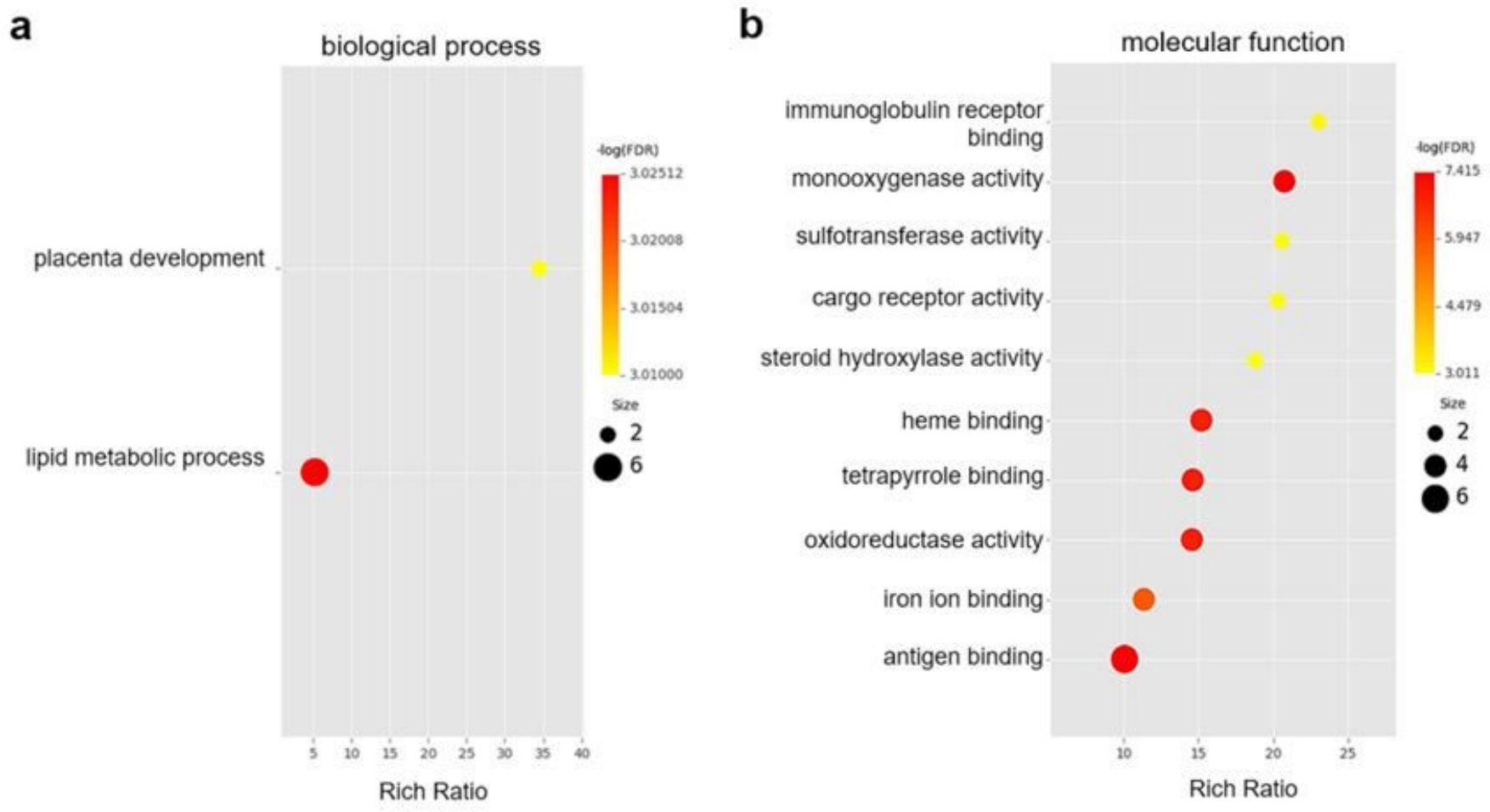

\section{Figure 5}

GO enrichment of each category: (a) Enrichment in biological process; (b) Top 10 enrichment terms in molecular function.

\section{Supplementary Files}

This is a list of supplementary files associated with this preprint. Click to download.

- SupplementaryMaterials.docx 